\title{
Uma análise histórica da construção de significados físicos para o conceito de potencial vetor no eletromagnetismo clássico+**
}

\author{
Aldo Aoyagui Gomes Pereira ${ }^{1}$ \\ Instituto Federal de Educação, Ciência e Tecnologia de São Paulo \\ Campus Piracicaba - SP \\ Cibelle Celestino Silva ${ }^{2}$ \\ Instituto de Física - Universidade de São Paulo \\ Campus de São Carlos - SP
}

\section{Resumo}

Atualmente o conceito de potencial vetor é geralmente tratado nos livrostexto e ensinado nos cursos universitários de eletromagnetismo como um artifício matemático para o cálculo dos campos elétrico e magnético. Porém, a investigação histórica da origem e desenvolvimento deste conceito, principalmente nos trabalhos de Michael Faraday e James Clerk Maxwell, nos deu indícios de que estes cientistas atribuíam significados físicos e análogos mecânicos a grandezas que atualmente recebem a denominação de potencial vetor. No contexto no qual estes cientistas trabalhavam, segunda metade de século XIX, a comunidade científica considerava que os fenômenos eletromagnéticos ocorriam em um éter com propriedades mecânicas e que as grandezas eletromagnéticas deveriam ter análogos mecânicos. No final deste mesmo século, alguns físicos, entre eles, Oliver Heaviside e Heinrich Hertz, reformularam a teoria de Maxwell, abandonando a interpretação física dada por Maxwell ao potencial vetor. Neste trabalho, discutimos sinteticamente como se deu esse processo de mudança. Para isso, realizamos um estudo histórico pautado em fontes primárias e secundárias sobre o assunto e, por último, investigamos a abordagem usada em alguns livros-texto de eletromagnetismo no ensino deste conceito. Apresentamos ainda, indícios de que o abandono

\footnotetext{
+ A historical analysis of the construction of physical meanings to the concept of vector potential in classical electromagnetism

* Recebido: abril de 2017. Aceito: julho de 2017.

${ }^{1}$ E-mail: agpereira980542@gmail.com, ${ }^{2}$ E-mail: cibelle@ifsc.usp.br
} 
da interpretação física ao conceito de potencial vetor esteve associado a posturas filosóficas e metodológicas, bem como ao interesse em solucionar problemas práticos, na recente indústria de cabos telegráficos na Grã-Bretanha do século XIX.

Palavras-chave: Eletromagnetismo Clássico; Potencial Vetor; História da Física; Ensino de Física.

\begin{abstract}
Currently, the concept of vector potential is usually treated in textbooks and taught in university courses of electromagnetism as a mathematical device for the calculation of electric and magnetic fields. However, the historical investigation of the origin and development of this concept, especially in the works of Michael Faraday and James Clerk Maxwell, gave us indications that these scientists attributed physical and mechanical analogical meanings to the quantities that currently receive the denomination of vector potential. In the context in which these scientists worked in the second half of the nineteenth century, the scientific community considered that electromagnetic phenomena occurred in an ether with mechanical properties and that electromagnetic quantities should have mechanical analogues. At the end of this century, some physicists, including Oliver Heaviside and Heinrich Hertz reformulated Maxwell's theory, abandoning the physical interpretation given by Maxwell to the vector potential. In this paper, we discuss in a syntactic way how this process of change occurred. For this, we conducted a historical study based on primary and secondary sources on the subject and, finally, investigated the approach used in some textbooks of electromagnetism in teaching this concept. We also present indications that the abandonment of physical interpretation of the concept of vector potential has been associated with philosophical and methodological positions as well as with the interest in solving practical problems in the recent telegraph cable industry in nineteenth-century Britain.
\end{abstract}

Keywords: Classical Electromagnetism; Vector Potential; History of Physics; Physics Teaching. 


\section{Introdução}

Quando estudamos eletromagnetismo nos cursos de física na universidade, é comum encontrarmos nos livros-texto afirmações do tipo: "como o campo elétrico é dito conservativo, ou seja, $\vec{\nabla} \times \vec{E}=0$, podemos associar uma função escalar $\varphi$ a ele da seguinte forma $\vec{E}=-\vec{\nabla} \varphi$ " (JACKSON, 1999, p. 30). A esta função $\varphi$ os livros dão o nome de potencial escalar e sua interpretação física está relacionada ao trabalho por unidade de carga, necessário para trazer uma carga do infinito até uma posição $\vec{r}$ qualquer em relação a um sistema de referência adotado. Em magnetostática não temos, em geral, o rotacional do campo magnético $\vec{B}$ nulo, então não podemos representá-lo, como o gradiente de uma função escalar. Mesmo nos casos em que há ausência de corrente, onde podemos considerar $\vec{\nabla} \times \vec{B}=0$, a introdução de um potencial escalar magnético esbarra em problemas teóricos, pois este não é univocamente determinado em um mesmo ponto do espaço (GRIFFITS, 1999, p. 239).

No caso do campo magnético, como seu divergente é sempre nulo, podemos representá-lo por um rotacional de uma função vetorial $\vec{A}$, uma vez que o divergente de um rotacional é nulo. A esta função vetorial damos o nome de potencial vetor. Ao contrário do potencial escalar, o potencial vetor parece não ter um significado físico consensual no eletromagnetismo clássico, sendo considerado como um artifício matemático para o cálculo dos campos elétrico e magnético (JACKSON, 1999, p. 236). No entanto, isto nem sempre foi assim. A investigação histórica sobre a origem do conceito nos trabalhos de Michael Faraday (1791-1867), Willian Thomson (1824-1907), James Clerk Maxwell (1831-1879), dentre outros, nos mostra que o papel do potencial vetor mudou bastante ao longo do desenvolvimento da teoria eletromagnética, sendo que na formulação final de Maxwell, ele desempenhou um papel importantíssimo até o final do século XIX, adquirindo um status semelhante ao conceito de momento na mecânica clássica.

Durante o século XIX, era comum na comunidade científica britânica, a utilização de modelos mecânicos e analogias no estudo dos fenômenos eletromagnéticos. O uso de analogias com fenômenos conhecidos, como o movimento dos fluidos, propagação de calor e estudo dos sólidos elásticos também era uma estratégia comum. Além disso, a existência de um éter permeando todo o espaço e responsável pelas interações e propagação dos fenômenos elétricos e magnéticos era praticamente um consenso entre os físicos britânicos. A explicação dos fenômenos eletromagnéticos era baseada na mecânica newtoniana e em termos de matéria, movimento e força no éter (SILVA, 2007). Atualmente não consideramos mais o éter eletromagnético como uma entidade física real. Apesar disso, aceitamos alguns dos resultados mais importantes da teoria eletromagnética criados supondo a existência do éter e baseados em uma abordagem mecânica do mesmo.

No final do século XIX, o físico-matemático Oliver Heaviside (1850-1925) introduziu a notação vetorial no eletromagnetismo escrevendo as equações de Maxwell na forma como as conhecemos hoje. Paralelamente, Heinrich Rudolf Hertz (1857-1894) realizou uma série de experimentos que culminaram na detecção de ondas eletromagnéticas em 1888. Neste contexto, 
tanto nos trabalhos de Heaviside quanto nos de Hertz, o significado físico do potencial vetor começou a perder importância. Ao contrário de Maxwell, estes físicos parecem não ter atribuído nenhum significado especial ao conceito de potencial vetor dentro da teoria eletromagnética clássica.

Neste trabalho, discutimos como se deu este processo de mudança dos papeis desempenhados pelo potencial vetor no eletromagnetismo clássico. Para isso, realizamos um estudo histórico pautado em fontes primárias e secundárias sobre o desenvolvimento do eletromagnetismo ao longo do século XIX, focando nossa atenção na abordagem de campo ${ }^{2}$. Por fim, analisamos alguns livros-texto de eletromagnetismo usados nos cursos de eletromagnetismo exemplificando algumas abordagens e posturas adotadas por estes autores sobre o ensino do conceito de potencial vetor.

\title{
II. O estado eletrotônico
}

Michael Faraday começou suas pesquisas em eletricidade por volta de 1821, influenciado principalmente, pelas então recentes experiências de Hans Christian Oersted (1777-1851) sobre relações entre eletricidade e magnetismo. Em novembro de 1825, o britânico iniciou uma série de experimentos que o levariam à explicação aceita atualmente sobre a indução eletromagnética. Após seis anos de experiências e estudos de trabalhos de outros cientistas da época, entre eles Joseph Henry (1797-1878) e Gerritt Moll (1785-1838), Faraday desenvolveu um novo equipamento que multiplicava o efeito da corrente produzida por espiras, utilizando um núcleo de ferro em forma de anel. Ao conectar o circuito secundário a um galvanômetro e o primário a uma bateria, ele notou que (FARADAY, 1839, p. 8):

\begin{abstract}
O galvanômetro é imediatamente afetado num grau muito maior do que em experimentos anteriores. Mas, embora o contato permanecesse ligado, o efeito não é permanente, pois a agulha do galvanômetro volta para sua posição inicial rapidamente, como se fosse indiferente ao efeito constante produzido pelo equipamento eletromagnético. Quando quebramos o contato com a bateria, a agulha é novamente defletida, mas agora em direção contrária à do exemplo anterior. [tradução nossa]
\end{abstract}

Nessa época, Faraday ainda não usava o conceito de linhas de força magnética, sendo sua primeira explicação para o fenômeno baseada no conceito de tensões ou estado de tensão assumido pelas partículas do circuito secundário quando da passagem da corrente no circuito primário. De acordo com ele, quando se liga o circuito primário, este induz uma corrente no secundário que dura enquanto a corrente no primário estiver aumentando. Atingido o valor

\footnotetext{
${ }^{2} \mathrm{Na}$ mesma época desenvolveu-se principalmente na Alemanha, uma teoria eletromagnética baseada no conceito de ação à distância. Ambas formulações do eletromagnetismo prediziam os resultados experimentais da época, diferenciando-se em seus aspectos filosóficos relacionados a interpretações de como se dão as interações na natureza (ASSIS, 1992).
} 
constante da corrente no primário, o secundário, apesar de não possuir mais uma corrente induzida, entraria num estado de tensão ou estado eletrotônico, no qual as partículas deste estão alinhadas na mesma direção da corrente induzida inicial. Ao desligar o primário, o estado de tensão é liberado por meio da produção de uma corrente induzida, agora no sentido contrário da corrente indutora inicial (FARADAY, 1839, p. 16).

Faraday não explicou detalhes sobre o que seria o estado eletrotônico, mas partiu do princípio de que ele estaria associado às partículas da matéria, seria perpendicular ao campo magnético local e paralelo à corrente que o produziu. Estas características, de acordo com Roche (1990), são semelhantes às propriedades qualitativas do conceito de potencial vetor. Por exemplo, quando pensamos no potencial vetor produzido por um fio longo a uma distância $r$ deste, ele possui a mesma direção da corrente que o produziu e é perpendicular ao campo magnético local.

\section{Origem e desenvolvimento do conceito de potencial vetor nos trabalhos de Maxwell}

Em 1861, Maxwell desenvolveu um modelo mecânico para o eletromagnetismo, atribuindo um significado mecânico para o conceito de estado eletrotônico introduzido anteriormente por Faraday. Desde 1835, em uma carta para Willian Thomson (1824-1907), Maxwell descreveu seu interesse pelo estudo deste conceito (MAXWELL apud BORK, 1967, p. 211):

\footnotetext{
Eu pretendo aplicar a estes fatos as noções de Faraday sobre o conceito de estado eletrotônico. Eu tenho trabalhado muito com ideias matemáticas e acredito ter conseguido muitas verdades e resultados relacionados com o conceito de estado eletrotônico. [tradução nossa]
}

O primeiro passo na concretização destas ideias só veio a se concretizar em 1856 num trabalho chamado On Faraday's lines of force.

\section{III.1 Intensidade eletrotônica}

Neste trabalho de 1856, Maxwell definiu a quantidade de corrente elétrica como sendo o que se conhece hoje por densidade de corrente elétrica $\vec{J}$, utilizando a notação $a_{2}, b_{2}$ e $c_{2}$ para as suas componentes. As letras gregas $\alpha_{2}, \beta_{2}$ e $\gamma_{2}$ denotavam as componentes da intensidade da força eletromotriz, cuja notação moderna é $\vec{E}$. Para o magnetismo, Maxwell usou os símbolos $a_{1}, b_{1}$ e $c_{1}$ para as componentes da quantidade de indução magnética ou corrente magnética, que, de acordo com suas equações, equivale ao conceito atual de campo magnético $\vec{B}$. Para as componentes da intensidade magnética, Maxwell usou os símbolos $\alpha_{1}, \beta_{1}$ e $\gamma_{1}$, que correspondem ao que atualmente é indicado pela letra $\vec{H}$. A tabela 1 apresenta estas quantidades e intensidades (vetores representam notação moderna, e componentes cartesianas, a representação de Maxwell). 
Tabela 1 - Relação entre grandezas elétricas e magnéticas de acordo com o artigo de 1856.

\begin{tabular}{ccc}
\hline & Intensidade & Quantidade \\
\hline Eletrotônica & $\vec{A}=\left(\alpha_{0}, \beta_{0}, \gamma_{0}\right)$ & - \\
\hline Magnética & $\vec{H}=\left(\alpha_{1}, \beta_{1}, \gamma_{1}\right)$ & $\vec{B}=\left(a_{1}, b_{1}, c_{1}\right)$ \\
\hline Elétrica & $\vec{E}=\left(\alpha_{2}, \beta_{2}, \gamma_{2}\right)$ & $\vec{J}=\left(a_{2}, b_{2}, c_{2}\right)$ \\
\hline
\end{tabular}

De acordo com Wise (1979), usando o conceito de linhas de força, desenvolvido por Faraday anos antes, Maxwell obteve um resultado semelhante ao obtido por André Marie Ampère (1775-1836) - hoje conhecida por lei circuital de Ampère. Maxwell associou linhas fechadas representando a quantidade elétrica $\vec{J}$ à produção de uma intensidade magnética $\vec{H}$ (WISE, 1979 , p. 1313). A produção de uma corrente fechada e, portanto, circular $\vec{J}$, tem como efeito a produção de uma intensidade magnética $\vec{H}$, também circular e perpendicular a $\vec{J}$. Matematicamente, significava que Maxwell poderia representar $\vec{J}$ como o rotacional de $\vec{H}$ (Fig. 1a).

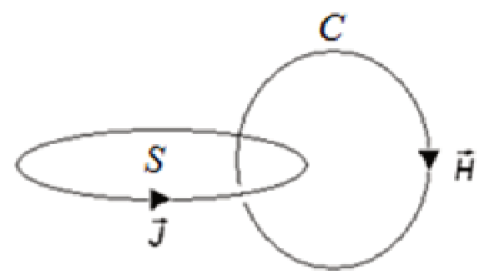

(a)

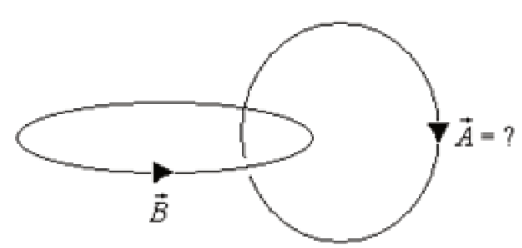

(b)

Fig. $1-A$ relação entre $\vec{J}$ e $\vec{H}$, como sugerido pela Fig. la, contribuiu para que Maxwell pensasse numa relação análoga entre $\vec{B}$ e $\vec{A}$, como na Fig. 1.b, dado que ambos $\vec{J} e$ $\vec{B}$ obedeciam uma condição solenoidal.

Para Maxwell, a soma da intensidade magnética em torno de uma curva fechada C é medida pela quantidade de corrente que passa através desta curva S (MAXWELL, 1965, p. 206). Podemos escrever esta afirmação em notação moderna como:

$$
\int_{C} \vec{H} \cdot d \vec{l} \propto \int_{S} \vec{J} \cdot d \vec{S}
$$

$d \vec{l}$ é o elemento de comprimento e $d \vec{S}$ o elemento de área do circuito fechado.

De acordo com Wise (1979), Maxwell raciocinou da seguinte forma: se uma quantidade de corrente elétrica produz uma intensidade magnética, seria possível uma quantidade magnética produzir uma intensidade elétrica? Ou, em linguagem moderna: já que uma quantidade de corrente elétrica $\vec{J}$ produz uma intensidade magnética $\vec{H}$, seria possível que uma quantidade magnética $\vec{B}$ produzisse uma intensidade elétrica, digamos $\vec{A}$ ? (Fig. 1a e 1b).

Maxwell deduziu uma equação, chamada por ele de equação da continuidade, relacionando as componentes da quantidade de corrente elétrica $a_{2}, b_{2}$ e $c_{2}$ da seguinte forma: (MAXWELL, 1965, p. 195): 


$$
\frac{d a_{2}}{d x}+\frac{d b_{2}}{d y}+\frac{d c_{2}}{d z}=0
$$

Podemos escrever esta relação em notação vetorial moderna como $\vec{\nabla} \cdot \vec{J}=0$. Assim, matematicamente falando, é possível escrever $\vec{J}$ como o rotacional de um vetor, neste caso $\vec{H}$ , já que a única condição matemática para isto é que $\vec{\nabla} \cdot \vec{J}=0$.

Já que a quantidade magnética $\vec{B}$ satisfaz uma condição análoga à equação (2), ou seja, $\vec{\nabla} \cdot \vec{B}=0$ (condição conhecida por Maxwell) então podemos escrevê-la como o rotacional de uma intensidade elétrica (WISE, 1979). Na interpretação de Wise, que também adotamos neste trabalho, Maxwell notou que o conceito de estado eletrotônico desenvolvido por Faraday alguns anos antes possuía propriedades semelhantes à intensidade elétrica aqui desenvolvida: dependia da quantidade magnética local, era perpendicular a esta, era criado e destruído de acordo com a criação e destruição desta quantidade magnética, e caracterizava-se por criar tensões no meio circundante. Maxwell associou então sua intensidade elétrica ao estado eletrotônico de Faraday, expressando-o matematicamente como $\vec{B}=\vec{\nabla} \times \vec{A}$ (WISE, 1979, p. 1315).

Em seguida, Maxwell enunciou uma lei análoga à equação (1) obtendo reciprocidade e simetria entre as curvas fechadas (MAXWELL, 1979, p. 206):

A intensidade eletrotônica total em torno do contorno de um elemento de superfície mede a quantidade de indução magnética que passa através desta superfície, ou, em outras palavras, o número de linhas de força magnética que passam através desta superfície. [tradução nossa]

Expressando em notação moderna:

$$
\int_{C} \vec{A} \cdot d \vec{l} \propto \int_{S} \vec{B} \cdot d \vec{S}
$$

$\vec{A}$ é a intensidade eletrotônica, cujas componentes, Maxwell escreveu como $\alpha_{0}, \beta_{0} \mathrm{e}$ $\gamma_{0}$ (Tabela 1). A partir da equação (3) é possível encontrar a relação entre intensidade eletrotônica e a força eletromotriz produzida pela ação de ímãs ou correntes ${ }^{3}$. Pela lei da indução de Faraday, podemos escrever:

$$
\int_{C} \vec{E} \cdot d \vec{l}=-\frac{d}{d t} \int_{S} \vec{B} \cdot d \vec{A}
$$

substituindo a integral do lado direito pela integral da equação (3) obtemos:

$$
\int_{C} \vec{E} \cdot d \vec{l}=-\frac{d}{d t} \iint_{C} \vec{A} \cdot d \vec{l}
$$

Como as integrais são calculadas sobre a mesma curva, obtemos a relação:

$$
\vec{E}=-d \vec{A} / d t
$$

\footnotetext{
${ }^{3}$ Maxwell encontrou estas relações utilizando leis oriundas do trabalho Conservation of Force de Hermann Von Helmholtz (1821-1894) (MAXWELL, 1965, p. 204). Neste trabalho, usamos o mesmo método de Darrigol (DARRIGOL, 2000, p. 146), utilizando notação vetorial moderna.
} 
Maxwell obteve, a menos de uma constante, as mesmas relações que, em sua notação são:

$$
\alpha_{2}=-\frac{1}{4 \pi} \frac{d \alpha_{0}}{d t}, \beta_{2}=-\frac{1}{4 \pi} \frac{d \beta_{0}}{d t} \text { e } \gamma_{2}=-\frac{1}{4 \pi} \frac{d \gamma_{0}}{d t}
$$

De acordo com a interpretação de Maxwell, as equações (6) relacionam a lei da indução de Faraday com a noção de estado eletrotônico. Neste momento, parece que Maxwell não atribuiu nenhum significado mecânico a este conceito, isto aconteceu somente em 1861, em seu trabalho On physical lines of force.

\section{III.2 A intensidade eletrotônica e a ação à distância}

Em uma carta para Thomson de 1854, Maxwell expressou algumas insatisfações em relação à explicação dada por Faraday sobre o efeito da indução eletromagnética produzido por circuitos (MAXWELL apud WISE, 1979, p. 1315):

[...] A força eletromotriz ao longo de uma linha la intensidade motora que tende a produzir uma corrente ao longo desta linhal é medida pelo número de linhas de força magnética que cortam aquela linha por unidade de tempo. Portanto, a força eletromotriz em torno de um dado circuito depende do decréscimo do número de linhas de força magnética que passam através do circuito por unidade de tempo. [tradução nossa]

Em outra carta para Thomson da mesma época, Maxwell expressou seu desconforto com o uso do conceito de linhas de força em situações envolvendo correntes fechadas (MAXWELL apud HARMAN, 1990, p. 16):

[...] Esta lei, embora simples e geral para tornar inteligível todos os fenômenos da indução em circuitos fechados, contém o conceito artificial do número de linhas de força passando através do circuito exercendo uma influência física sobre ele. Seria melhor se nós pudéssemos evitar, no enunciado desta lei, o uso deste conceito artificial. [tradução nossa]

As preocupações de Maxwell se justificam, pois, de acordo com a visão da época, se os fenômenos eletromagnéticos fossem intermediados por um meio, haveria um problema neste enunciado, que foi reconhecido por Faraday bem antes de Maxwell (WISE, 1979). Como é possível uma quantidade magnética afetar sua força eletromotriz simplesmente passando através do circuito, sem entrar em contato com este? Por exemplo, imagine um solenoide percorrido por uma corrente elétrica variável e que, portanto, induz uma força eletromotriz num circuito próximo, como mostrado na Fig. 2. De acordo com Maxwell e Faraday, a corrente induzida $\boldsymbol{I}_{\text {ind }}$ é produzida pela passagem das linhas de força magnética representadas por $\vec{B}$ (supondo que a 
corrente indutora $\boldsymbol{I}$ seja variável). Como as linhas de força magnética não tocam o circuito externo, seria necessário supor uma ação à distância para explicar a existência da corrente neste circuito (LORENZO, 1995).

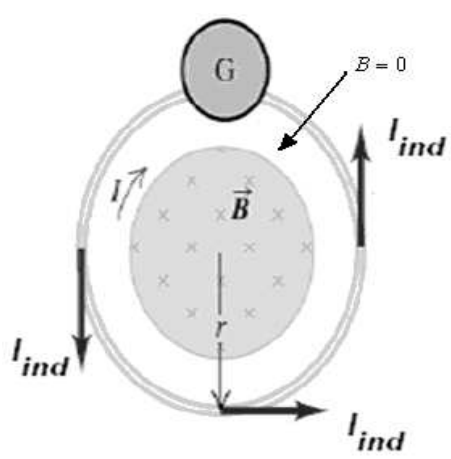

Fig. 2 - De acordo com a citação acima, usar o conceito de linhas de força magnética para explicar a indução eletromagnética levaria ao uso do conceito de ação à distância no caso da figura acima. Tendo em vista que o conceito de ação à distância era inaceitável entre a maioria dos físicos britânicos, isto era um problema.

Para contornar este problema, Maxwell usou o conceito de intensidade eletrotônica, como empregado nas equações (6), pois de acordo com ele, o problema da ação à distância na indução eletromagnética estaria resolvido (MAXWELL, 1965, p. 203):

[...] nós obtemos agora, com as funções $\alpha_{0}, \beta_{0}$ e $\gamma_{0}$, os meios de evitar a consideração da quantidade de indução magnética que passa através do circuito. Em vez deste método artificial, nós usamos um mais natural, que é considerar a corrente com referência a quantidades existindo no mesmo espaço que ela própria. A estas funções, eu dou o nome de funções eletrotônicas, ou componentes da intensidade eletrotônica. [tradução nossa]

Acreditamos que dentro do programa de explicações mecânicas dos fenômenos eletromagnéticos, Maxwell esperava desenvolver uma interpretação mecânica para o conceito de estado eletrotônico. Ele declarou sua insatisfação ao usar apenas símbolos matemáticos para descrever uma grandeza tão importante quanto o estado eletrotônico. No final da primeira parte do artigo de 1856, ele deixou claro suas intenções para um projeto futuro (MAXWELL, 1965, p. 188): "Através de um estudo cuidadoso das leis dos sólidos elásticos e do movimento dos fluidos viscosos, eu espero descobrir um método de formar uma concepção mecânica para o conceito de estado eletrotônico adaptado a um tipo de raciocínio geral”.

Este objetivo foi alcançado com o desenvolvimento de um modelo mecânico para explicar as interações elétricas e magnéticas no fenômeno da indução eletromagnética baseado na existência de vórtices no éter. 


\section{III.3 Momento reduzido}

Durante o século XIX, era comum entre os físicos britânicos o uso de modelos e analogias para explicar os fenômenos físicos. Nesta tradição, Maxwell procurou análogos mecânicos para as grandezas eletromagnéticas, inclusive para a intensidade eletrotônica. No artigo $O n$ physical lines of force, publicado na Philosophical Magazine em 1861, Maxwell explorou mecanismos capazes de explicar a transmissão do efeito elétrico entre condutores no fenômeno da indução eletromagnética. De acordo com Darrigol (2000, p. 150), ele foi influenciado por trabalhos de mecânica sobre máquinas que usavam rodas dentadas e alavancas em seu funcionamento, entre eles o livro de Willian John Macquorn Rankine (1820-1872) Applied mechanics, de 1858 .

Maxwell representou o éter como um meio constituído de células como se fosse uma colmeia formada por vórtices de formato hexagonal e elástico girando em torno do próprio eixo (Fig. 3a). O modelo tinha como elemento principal vórtices adjacentes girando no mesmo sentido, e pequenas partículas esféricas intermediárias (idle wheels) que possibilitavam o movimento no mesmo sentido dos vórtices adjacentes. Como a velocidade angular de vórtices adjacentes é diferente, as esferas entre os vórtices adquirem, além de um movimento de rotação, um de translação, provocando um fluxo de partículas que Maxwell associou à corrente elétrica (Fig. $3 b)$.

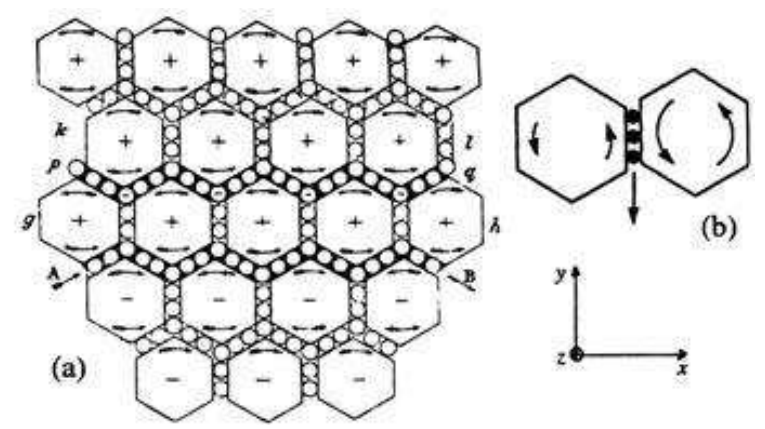

Fig. 3 - Modelo mecânico representado pelos vórtices e esferas intermediárias (FONTE: DARRIGOL, 2000, p. 150).

O torque produzido por esta força é proporcional à derivada temporal do momento angular da célula, que estaria associado à rotação dos vórtices, e proporcional à intensidade da força magnetizadora. Além disso, pelo princípio da ação e reação, o torque exercido pelas esferas nos vórtices deveria ser igual e oposto à derivada temporal do momento angular produzido pelos vórtices. Utilizando este modelo mecânico, Maxwell deduziu as relações abaixo (MAXWELL, 1965, p. 475):

$$
\frac{d Q}{d z}-\frac{d R}{d y}=\mu \frac{d \alpha}{d t}, \frac{d R}{d x}-\frac{d P}{d z}=\mu \frac{d \beta}{d t} \mathrm{e} \frac{d P}{d y}-\frac{d Q}{d x}=\mu \frac{d \gamma}{d t} .
$$


Aqui, Maxwell mudou a notação em relação ao artigo de 1856. Em vez de usar $\alpha_{2}$, $\beta_{2}$ e $\gamma_{2}$ para as componentes da força eletromotriz, ele usou $P, Q$ e $R$. E ao invés de $\alpha_{1}, \beta_{1}$ e $\gamma_{1}$ para as componentes da intensidade da força magnética, usou $\alpha, \beta$ e $\gamma$. As equações (7) podem ser escritas em notação vetorial moderna como:

$$
\vec{\nabla} \times \vec{E}=-\mu \frac{\partial \vec{H}}{\partial t} .
$$

Integrando ambos os lados da equação (8) numa superfície qualquer, e usando o teorema integral de Stokes e a relação $\vec{\nabla} \times \vec{A}=\vec{B}^{4}$, encontramos a relação entre intensidade eletrotônica e força eletromotriz: $\vec{E}=-d \vec{A} / d t$. Para as componentes da intensidade eletrotônica, ele usou as letras $F, G$ e $H^{5}$. Assim, podemos escrever os resultados obtidos na notação de Maxwell (MAXWELL, 1965, p. 476) como:

$$
P=\frac{d F}{d t}, Q=\frac{d G}{d t} \text { e } R=\frac{d H}{d t} .
$$

O objetivo de Maxwell, enunciado no final do artigo de 1856, parecia agora estar bem próximo de ser alcançado. Após a dedução das equações (9), ele escreveu (MAXWELL, 1965, p. 476):

Nós temos agora determinado três quantidades, $F, G$ e $H$, das quais podemos determinar $P, Q$ e $R$ por meio da consideração de como as primeiras variam em relação ao tempo. Num artigo anterior, eu tive razões para considerar as quantidades $F, G e$ H como as componentes daquilo que Faraday tinha conjecturado existir, e chamei de estado eletrotônico. Naquele artigo, deduzi as relações matemáticas entre o estado eletrotônico e as linhas de força magnética, como expresso nas equações (55), e também as relações entre estado eletrotônico e a força eletromotriz expressas nas equações (58). Nós vamos agora nos aventurar a interpretar estas quantidades de um ponto de vista mecânico ${ }^{6}$. [tradução nossa]

O movimento giratório adquirido pelos vórtices em seu modelo mecânico e as equações (9) sugeriram a Maxwell uma analogia entre grandezas mecânicas e elétricas. Para ele, a força eletromotriz num dado ponto é obtida pela derivada temporal das componentes da intensidade eletrotônica no mesmo ponto. Como, pela segunda lei de Newton, a força em determinado ponto é determinada pela derivada temporal do momento neste ponto, Maxwell associou as componentes da intensidade eletrotônica a algum tipo de momento localizado no meio, ao

\footnotetext{
${ }^{4}$ Maxwell atribuiu esse teorema a Stokes contido na obra On the dynamical theory of diffraction de 1849. A relação $\vec{\nabla} \times \vec{A}=\vec{B}$ já havia sido deduzida por Maxwell no artigo de 1856 .

${ }^{5}$ Esta mudança de notação tanto para a força eletromotriz como para a intensidade eletrotônica, pode ser devido à leitura do trabalho de Thomson de 1851, A mathematical theory of magnetism, que utiliza a notação $F, G, H$ num contexto matemático semelhante ao apresentado aqui (THOMSON, 1851).

${ }^{6}$ As equações (55) e (58) às quais ele se refere são, em uma notação moderna, $\vec{\nabla} \times \vec{A}=\vec{B}$ e $\vec{E}=-\frac{d \vec{A}}{d t}$, respectivamente.
} 
qual ele chamou de momento reduzido. Assim, as componentes $F, G$ e $H$, corresponderiam ao impulso que agiria no eixo de uma roda de uma máquina se a velocidade com que ela gira fosse adquirida instantaneamente, estando ela inicialmente em repouso (MAXWELL, 1965, p. 478).

Comparando as equações (9) (em notação vetorial moderna) com a segunda lei de Newton, é possível associar $\vec{F} \operatorname{com} \vec{E}$ e $\vec{p} \operatorname{com} \vec{A}$, ou seja, $\vec{F}=\frac{d \vec{p}}{d t} \Rightarrow \vec{E}=-\frac{d \vec{A}}{d t}$. Nas palavras de Maxwell (1965, p. 479):

\begin{abstract}
[...] O impulso de uma máquina pode ser calculado em qualquer parte do mecanismo, e deve ser chamado de momento reduzido da máquina naquele ponto. Ao variar o movimento desta máquina, a força em qualquer parte, devido à variação deste movimento, pode ser encontrada diferenciando o momento reduzido em relação ao tempo, assim, para encontrarmos a força eletromotriz num sistema de vórtices por meio do estado eletrotônico, devemos realizar o mesmo processo. [tradução nossa]
\end{abstract}

Desta forma, Maxwell interpretou o potencial vetor como um análogo mecânico ao estado eletrotônico de Faraday, chamando-o de momento reduzido do sistema de vórtices, sendo a variação desta grandeza num determinado ponto igual a força eletromotriz neste ponto.

\title{
III.4 Os quaternions e o nome potencial vetor
}

Em 1867, Maxwell começou a estudar o livro publicado recentemente por Peter Guthrie Tait (1831-1901, An Elementary treatise on quaternions (SILVA, 2002). Nesse mesmo ano, ele começou a escrever o livro A Treatise on electricity and magnetism, publicado seis anos depois ${ }^{7}$. Além de apresentar suas próprias ideias sobre a teoria eletromagnética, o livro também foi pensado para ser usado como guia para alunos e professores nas universidades, principalmente na Universidade de Cambridge. O conceito chamado de momento reduzido no artigo de 1861, passa a ser chamado de potencial vetor neste livro. Como descrevemos abaixo, apresentamos alguns indícios de que a mudança no nome pode ser relacionada ao contato de Maxwell com a álgebra quaterniônica.

Os quaternions são entes matemáticos formados por quatro números: um escalar e três componentes de um vetor, formando uma álgebra não comutativa (SILVA; MARTINS, 2002). Podemos representá-los simbolicamente por $q=a+i x+j y+k z$, em que $a$ representa a parte escalar do quaternion, $x, y, z$ as componentes da parte vetorial e $i, j, k$, vetores unitários. As leis da álgebra vetorial atual são contempladas, incluindo as operações usuais de soma de vetores, os produtos escalares e vetoriais, o operador $\nabla$ (aqui chamado de operador de Hamilton), os teoremas de Gauss e Stokes e a funções lineares vetoriais. As semelhanças entre quaternions e vetores são grandes, mas existem algumas diferenças importantes. Por exemplo, para os vetores unitários $i, j, k$ de um quaternion valem as seguintes propriedades:

\footnotetext{
${ }^{7}$ No presente trabalho nos referimos a esta obra como Treatise.
} 


$$
i^{2}=j^{2}=k^{2}=-1, j k=-k j=i, k i=-i k=j \text { e } i j=-j i=k,
$$

já que são anticomutativos.

Tait também definiu o operador $\nabla$, batizado por Maxwell como nabla, que aplicado a um quaternion resulta em outro quaternion. Escrevendo $q=a+i F+j G+k H$, então a operação $\nabla q$ resulta em:

$$
\nabla q=\left(i \frac{d a}{d x}+j \frac{d a}{d y}+k \frac{d a}{d z}\right)-\left(\frac{d F}{d x}+\frac{d G}{d y}+\frac{d H}{d z}\right)+\left(\frac{d H}{d y}-\frac{d G}{d z}\right) i+\left(\frac{d F}{d z}-\frac{d H}{d x}\right) j+\left(\frac{d G}{d x}-\frac{d F}{d y}\right) k
$$

O termo potencial vetor é usado pela primeira vez no artigo 405, intitulado The vector potential of magnetic induction, do livro A Treatise on electricity and magnetism de Maxwell. Neste artigo, Maxwell calculou a indução magnética através de uma curva fechada por um processo dependendo somente da natureza da curva, sem envolver processos dependentes da construção de uma superfície formada sobre esta curva. Ele inferiu que isto é possível desde que encontremos um vetor $U^{8}$ relacionado à indução magnética $B$, de tal forma que a integral de linha de $U$ ao longo da curva fechada fosse igual à integral de superfície de $B$ sobre toda a superfície fechada (MAXWELL, 1954, p. 29, v. 2). Ele, então, concluiu: "O vetor $U$, cujas componentes são $F, G$ e $H$, é chamado de potencial vetor da indução magnética". Ele não faz menção deste conceito em trabalhos anteriores, nem relaciona o momento reduzido do trabalho de 1861 a este potencial vetor da indução magnética. Apesar de Maxwell não esclarecer explicitamente a mudança de nome, a análise de algumas operações quaterniônicas dá uma pista para esta mudança.

Admitindo que $U$ seja um vetor puro, ou seja, um quaternion com a parte escalar nula, com componentes $F, G$ e $H$, ao realizarmos o produto do operador $\nabla$ por $U$ obtemos, assim como em (11)16:

$$
B=-\left(\frac{d F}{d x}+\frac{d G}{d y}+\frac{d H}{d z}\right)+\left(\frac{d H}{d y}-\frac{d G}{d z}\right) i+\left(\frac{d F}{d z}-\frac{d H}{d x}\right) j+\left(\frac{d G}{d x}-\frac{d F}{d y}\right) k
$$

onde $B$ é o quaternion indução magnética e $i, j, k$, vetores unitários.

A parte escalar do quaternion $B$ em (12) é nula ou, usando uma linguagem moderna, devido à condição $\vec{\nabla} \cdot \vec{U}=0$, usada por Maxwell tanto no Treatise quanto no artigo de 1861 . Então o que sobra é a parte vetorial de (12) que, neste caso, nada mais é do que $\vec{B}=\vec{\nabla} \times \vec{U}$ em notação moderna. Assim, ao usar o operador $\nabla$ para obter a indução magnética a partir do quaternion $U$, que era a operação usual nesta época para encontrar a força por meio de um potencial qualquer, Maxwell deduziu que a indução magnética $B$ pode ser escrita como o rotacional de seu potencial vetor, como fica claro na citação (MAXWELL, 1954, p. 31-32, v. 2):

\footnotetext{
8 Neste livro Maxwell utilizou letras góticas alemãs, usamos ao longo deste trabalho as mesmas letras que Maxwell, porém na mesma fonte do texto deste trabalho.
} 
Nós vimos que a força magnética $H$ pode ser encontrada de seu potencial escalar magnético V pela aplicação do operador de Hamilton $\nabla$, tal que nós podemos escrever $H=-\nabla V$, esta equação é verdadeira, tanto dentro quanto fora de ímãs. Da presente investigação parece que a indução magnética B pode ser calculada por meio de seu potencial vetor U pela aplicação do mesmo operador, e o resultado é verdadeiro tanto dentro quanto fora dos ímãs. A aplicação deste operador a uma função vetorial produz, em geral, uma quantidade escalar e uma vetorial. A parte escalar, no entanto, que nós temos chamado de convergência da função vetorial, neste caso satisfaz a condição solenoidal

$\frac{d F}{d \xi}+\frac{d G}{d \eta}+\frac{d H}{d \zeta}=0$

Nós podemos, portanto, escrever a relação entre a indução magnética e seu potencial vetor como $B=\nabla U$, que pode ser expressa em palavras, dizendo que a indução magnética é o rotacional de seu potencial vetor.

Desta citação vê-se que o novo nome deriva diretamente da utilização da notação quaterniônica. Em geral, na álgebra quaterniônica, $B=\nabla U$ e $B=\nabla \times U$ não são iguais. Esta igualdade depende da condição auxiliar que estabelecemos para $U$, neste caso $\vec{\nabla} \cdot \vec{U}=0$.

Nos anos seguintes, este conceito adquiriu uma importância fundamental na teoria de Maxwell, sendo interpretado como uma grandeza que permitia, entre outras coisas, explicar as interações eletromagnéticas entre corpos carregados sem considerar ação à distância.

\section{Hertz, os maxwellianos e o conceito de potencial vetor}

No final do século XIX, alguns físicos, entre eles Oliver Heaviside (1850-1925) e Heinrich Rudolf Hertz (1857-1894), passaram a questionar o status de grandeza fundamental atribuída por Maxwell ao potencial vetor. Eles passaram a enfatizar o papel representado pelos campos $\vec{E}$ e $\vec{H}$, eliminando os potenciais das equações principais do eletromagnetismo.

Na década de 1880 , o eletromagnetismo era uma teoria que ainda estava sendo construída e não havia consenso sobre seus aspectos fundamentais. A teoria desenvolveu-se em dois segmentos independentes: a teoria eletromagnética desenvolvida no continente, cujos nomes mais proeminentes foram Franz Ernst Neumann (1798-1895), Wilhem Weber (1804-1891) e Hermann von Helmholtz (1821-1894) e a teoria eletromagnética desenvolvida pelos britânicos, discutida acima. Os primeiros supunham que a interação eletromagnética entre os corpos ocorria instantaneamente à distância. Já para os britânicos, a interação eletromagnética era intermediada por um meio e levava tempo para se propagar. Apesar das duas teorias possuírem bases matemáticas estabelecidas e fazerem predições experimentais semelhantes, havia aspectos fundamentais a serem resolvidos. 
A decisão entre as duas teorias estava alicerçada em suas consequências empíricas. De acordo com a teoria de Maxwell, a mudança na polarização dielétrica produziria efeitos eletromagnéticos mensuráveis da mesma forma que as correntes convencionais produzem. Helmholtz procurava um teste experimental da existência destes efeitos, entre eles a produção de ondas eletromagnéticas por meio da polarização elétrica. Em 1879, ele propôs para a Academia de Ciências de Berlim um prêmio para quem realizasse um experimento capaz de decidir entre as duas formulações da teoria (MCCORMMACH, 1981).

Hertz começou a estudar o problema a partir de 1880, quando era assistente de Helmholtz. Em 1883, sob a indicação de Gustav Robert Kirchhoff (1824-1887), Hertz obteve o cargo de Privatdozent na Universidade de Kiel. Provavelmente devido à ausência de laboratórios bem equipados em Kiel, Hertz começou a aprofundar os seus estudos teóricos sobre os trabalhos de Maxwell. Influenciado pela busca da unidade entre as forças da natureza, assim como Oersted $^{9}$, Hertz procurou unificar os conceitos de força elétrica produzida por cargas eletrostáticas e por campos magnéticos variáveis (MCCORMMACH, 1981).

Por meio de operações matemáticas que fogem ao escopo deste trabalho, ele chegou à conclusão de que o formalismo adotado por Maxwell era compatível com as suposições teóricas de ambas as teorias eletromagnéticas: a britânica, baseada na interação intermediada por campos e a continental, que supunha a interação à distância. Além disso, Hertz percebeu que a força elétrica gerada por um campo magnético variável era idêntica, em sua natureza, à força elétrica produzida por cargas eletrostáticas, desde que o potencial vetor e escalar fossem eliminados da teoria de Maxwell (WHITTAKER, 1973).

Por um raciocínio de aproximações sucessivas, Hertz foi capaz de chegar às mesmas equações que Heaviside havia chegado em 1884 (WHITTAKER, 1973). Considerando as variações de corrente originadas num sistema contendo apenas correntes, ele obteve as seguintes equações (em notação moderna):

$$
\vec{\nabla} \times \vec{H}=\frac{1}{c} \frac{\partial \vec{E}}{\partial t}+\frac{4 \pi}{c} \vec{s} \text { e } \vec{\nabla} \times \vec{E}=-\frac{1}{c} \frac{\partial \vec{H}}{\partial t},
$$

onde $\vec{E}$ e $\vec{H}^{10}$ são os campos elétrico e magnético, respectivamente, e $\vec{s}$ é a densidade de corrente elétrica.

Ao retomar os seus trabalhos experimentais em 1886, Hertz convenceu-se de que apesar das teorias britânicas e continentais terem predições experimentais semelhantes, a teoria de

\footnotetext{
${ }^{9}$ No início do século XIX, os físicos que defendiam o modo de pensar baseado no Naturphilosophie eram definidos como uma comunidade de pessoas distintas da comunidade normal de físicos tradicionais da época. Eles eram intitulados filósofos da natureza, e defendiam que o dualismo era o princípio fundamental da física e da química. As influências do Naturphilosophie na descoberta do eletromagnetismo podem ser encontradas no trabalho de Martins (1986).
}

${ }^{10}$ Hertz não usava notação vetorial, ele escreveu as equações em componentes. 
Maxwell previa de forma mais direta e simples a propagação de ondas eletromagnéticas, consequência que só podia ser obtida da teoria continental através de artifícios matemáticos bem mais elaborados (ABRANTES, 1992).

Com a verificação experimental da teoria de Maxwell em 1888, Hertz propôs uma reconstrução axiomática da teoria de Maxwell, por considerá-la inaceitável na sua formulação original, baseada na necessidade da existência de um meio responsável pela propagação dos fenômenos elétricos e magnéticos e na predominância do conceito de potencial vetor e escalar nas principais equações. A partir disso, Hertz resumiu a teoria de Maxwell a somente quatro equações fundamentais, tomando o cuidado de apresentá-las como "relações entre magnitudes físicas que são efetivamente observadas, e não entre magnitudes que servem somente ao cálculo" (HERTZ, 1962, p. 196). Estre estas magnitudes que servem somente ao cálculo está o potencial vetor (HERTZ, 1962, p. 196):

Como uma ideia matemática de natureza rudimentar, eu devo mencionar a predominância do potencial vetor nas equações fundamentais. Na construção da minha nova teoria, o potencial serve apenas como material provisório; quando da introdução dos potenciais, as forças à distância, que apareciam descontinuamente em pontos particulares, foram trocadas por magnitudes que em cada ponto no espaço eram determinadas utilizando somente condições em pontos vizinhos. Mas, depois que aprendemos a ver as forças como magnitudes do tipo mencionado anteriormente, não há objeção em usá-las em vez dos potenciais, a menos que alguma vantagem matemática seja ganha no uso dos potenciais. E não parece para mim que qualquer vantagem é alcançada pela introdução do potencial vetor nas equações fundamentais. [tradução nossa]

As descobertas de Hertz e suas consequências para a teoria de Maxwell foram amplamente debatidas em um encontro promovido pela British Association of Science na cidade de Bath, na Inglaterra, em 1888, do qual participaram os principais pesquisadores sobre eletromagnetismo da época. Entre os temas discutidos neste encontro, figuravam a propagação dos potenciais e o abandono do conceito de potencial vetor.

Na teoria de Maxwell, o potencial escalar propagava-se instantaneamente devido à escolha do calibre de Coulomb para o potencial vetor. No entanto, John Henry Poynting (18521914) já havia demonstrado em 1884 que a explicação de como a energia se propagava no campo eletromagnético dependia apenas das densidades de energia elétrica e magnética, expressas por $\frac{1}{2} \varepsilon \vec{E}^{2}$ e $\frac{1}{2} \mu \vec{H}^{2}$. Ele calculou como estas grandezas se alteram dentro de um volume específico e concluiu que a taxa na qual a energia entra em uma determinada região, sendo armazenada ou dissipada como calor, depende exclusivamente dos campos $\vec{E}$ e $\vec{H}$, sendo o vetor que localiza e direciona o fluxo desta energia dado por $\vec{S}=\vec{E} \times \vec{H}$, hoje conhecido como vetor de Poynting (POYNTING, 1884).

Ao estudar o trabalho de Poynting, George Francis Fitzgerald (1851-1901) elaborou um modelo mecânico próprio para o éter na tentativa de explicar como se dava a propagação 
das ondas eletromagnéticas (DARRIGOL, 2000, p. 185]. Uma das consequências deste modelo, segundo Fitzgerald, era que não poderia haver nenhuma grandeza no eletromagnetismo que se propagasse instantaneamente. Ao invés de usar a condição usada por Maxwell em seu Treatise $\vec{\nabla} \cdot \vec{A}=0$, ele usou $\vec{\nabla} \cdot \vec{A}=-d \varphi / d t$, condição já usada pelos continentais, chegando a uma equação de onda para o potencial escalar (HUNT, 1991, p. 118):

$$
\nabla^{2} \varphi-\frac{d^{2} \varphi}{d t^{2}}=0
$$

Este resultado obtido por Fitzgerald contribuiu para um acirramento na discussão sobre quais grandezas se propagavam: os campos ou os potenciais. É interessante notar que, mesmo deduzindo uma equação de propagação para o potencial escalar, Fitzgerald adotou uma postura de usá-lo, assim como o potencial vetor, apenas como auxiliares para encontrar as grandezas que, de acordo com ele, seriam as dotadas de interesse físico e experimental: os campos $\vec{E}$ e $\vec{H}$. Para Fitzgerald, os 'potenciais eram 'malabarismos de cálculo' que, às vezes, obscurecem a realidade física de como a energia é localizada e propagada num campo eletromagnético" (FITZGERALD apud HUNT, 1991, p. 118).

A discussão sobre a propagação do potencial vetor parece não ter se aprofundada no encontro de Bath em 1888. Porém, quando Sir Oliver Lodge (1851-1940) publicou o resumo sobre as discussões envolvendo a propagação do potencial escalar, Heaviside, que não esteve presente no encontro, argumentou incisivamente que a extinção do conceito de potencial escalar não era suficiente. Em uma carta para Lodge, de 9 de outubro de 1888, Heaviside declarou que seu objetivo não era “... simplesmente assassinar o potencial escalar de Maxwell, mas também, aquele maravilhoso monstro de três pernas com um parasita escalar em suas costas, o assim chamado momento eletrocinético em um ponto" (HEAVISIDE apud HUNT, 1991, p. 166) ${ }^{11}$.

É importante lembrar que estas pesquisas e debates foram realizados em um contexto no qual os principais problemas práticos do eletromagnetismo na época estavam relacionados à detecção de ondas eletromagnéticas previstas pela teoria de Maxwell e a compreensão do comportamento da energia nas ondas eletromagnéticas transmitidas e recebidas por cabos telegráficos. Buscava-se compreender como transmitir a energia de forma limpa e sem distorções através de cabos telegráficos. Neste período não se conheciam os mecanismos pelos quais os fios transmitiam e controlavam o fluxo de energia em circuitos telegráficos. Para Heaviside, a resposta para esse problema estaria no Treatise de Maxwell. Ele considerava o livro de Maxwell de difícil compreensão, devido, entre outros fatores, ao fato de Maxwell escrever as equações do eletromagnetismo em componentes cartesianas e na notação dos quaternions (SILVA, 2002).

A representação do fluxo de energia em função dos campos, e não mais dos potenciais, começou a ser discutida por Heaviside entre 1883 e 1884, numa série de pequenos artigos publicados na revista semanal britânica The Electrician, e posteriormente reunidos no artigo intitulado The energy of electric currents. Neste trabalho, ele explicitou as vantagens de escrever

\footnotetext{
11 Momento eletrocinético era um dos nomes que Maxwell deu para o potencial vetor no livro A Treatise on Electricity and Magnetism.
} 
a energia no campo magnético devido a correntes, utilizando o campo magnético para expressála, e não o potencial vetor (HEAVISIDE, 1970, p. 249, v. 2):

Evidentemente, então, a expressão $\sum \frac{1}{2} A C$ não localiza a energia devidamente. Note também que, embora o total seja positivo, a porção referente a $\frac{1}{2} \mathrm{AC}$ em qualquer unidade de volume particular, pode ser positiva ou negativa, dependendo da direção da corrente e do potencial vetor. Nós devemos, portanto, descartar a ideia de que $\frac{1}{2} A C$ representa a energia por unidade de volume ou a densidade de energia. Mas nós temos a forma $\sum B^{2} / 8 \pi$ à disposição. Ela é idêntica à quantidade $\sum \frac{1}{2} A C$, mas indica uma distribuição diferente de energia. [tradução nossa]

Maxwell já havia escrito a energia devido a correntes como $\frac{1}{2} A \cdot C$, em que $A$ é o potencial vetor e $C$ a densidade de corrente (MAXWELL 1954, p. 273, v. 2). Porém, como apontou Heaviside, esta forma de expressar a energia contém sérios problemas. Um deles é o fato de que a energia, em qualquer ponto, depende não apenas da corrente neste ponto, mas também do potencial vetor neste ponto. O potencial vetor, por sua vez, depende do estado do sistema inteiro e não apenas do estado naquele ponto. A inconsistência está no fato de que a energia, seja potencial ou cinética, em um determinado ponto do campo, dependerá dos deslocamentos ou movimentos considerados naquele ponto e não do estado do sistema inteiro (HEAVISIDE, 1971, p. 249, v. 1).

Heaviside passa, então, a expressar a energia por $B^{2} / 8 \pi$, que para ele "expressa o fato de que a energia em qualquer ponto do campo depende somente do quadrado da força magnética naquele ponto, e não do estado de todo o resto do sistema" (HEAVISIDE, 1971, p. 249, v. 1).

Ao considerar como a energia se transmite pelo espaço, Heaviside concluiu que poderia escrever o fluxo de energia utilizando $\vec{S}=\vec{E} \times \vec{H}$, assim como Poynting havia feito anteriormente. Ou seja, o fluxo de energia num determinado ponto do espaço é simplesmente o produto vetorial dos campos elétrico e magnético neste ponto. Ao considerar esta equação como uma das fundamentais da teoria de Maxwell, Heaviside passou a empreender um método de obtê-la diretamente das equações básicas do campo elétrico e magnético, sem a utilização do potencial vetor (HUNT, 2012). Ele publicou o trabalho contendo estas novas equações em 1885 no The Electrician. A esta forma de escrever as equações de Maxwell, ele deu o nome de método duplex, organizando-as em quatro equações, de forma semelhante a que escrevemos atualmente (HEAVISIDE, 1970, p. 447, v. 1):

$$
\begin{gathered}
\vec{\nabla} \cdot \varepsilon \vec{E}=0, \vec{\nabla} \cdot \mu \vec{H}=0, \\
\vec{\nabla} \times \vec{H}=\varepsilon \frac{\partial \vec{E}}{\partial t} \text { e } \vec{\nabla} \times \vec{E}=-\mu \frac{\partial \vec{H}}{\partial t}
\end{gathered}
$$

Heaviside considerou estas quatro equações simples e simétricas em relação aos campos $\vec{E}$ e $\vec{H}$, sendo suficientes para o tratamento da propagação das ondas eletromagnéticas em função de grandezas que possuem significação física e, portanto, útil para os problemas práticos 
da época (HUNT, 2012). Ele considerou esta reconstrução da teoria de Maxwell uma de suas contribuições fundamentais para o eletromagnetismo, enfatizando em toda a sua obra a importância do abandono dos potenciais, principalmente pelo fato destes não possuírem um significado físico claro.

De acordo com ele, os potencias seriam vestígios de uma teoria de ação à distância, eles eram "funções vetoriais que não possuem nenhuma semelhança com os verdadeiros vetores que representam o estado físico do campo" (HEAVISIDE, 1971, p. 127, v. 1). Uma vez descartados estes vetores (HEAVISIDE apud O'HARA, PRICHA, 1987, p. 58):

\begin{abstract}
Muito da metafísica desaparece. Além disto, problemas que eram inicialmente obscuros e não manipuláveis quando expressos em função dos potenciais, podem agora ser facilmente resolvidos. Condições de contorno são, agora, autoevidentes e os trabalhos de cálculos são mais simples e claros. Importantes propriedades que eram anteriormente escondidas da visão pela intervenção dos potenciais, são agora facilmente reconhecidas. [tradução nossa]
\end{abstract}

Heaviside foi o primeiro a afirmar que a simetria das quatro equações deduzidas independentemente por ele e por Hertz, poderia ser interpretada como um conceito fundamental da teoria eletromagnética. Seus artigos posteriores foram uma campanha pela adoção destas equações, mostrando sua aplicabilidade para a solução de diversos problemas práticos e teóricos. Além disso, com severas críticas ao uso do potencial vetor, Heaviside frequentemente ridicularizava os físicos que o usavam. Isto contribuiu para que diversos físicos do final do século XIX e início do XX, começassem a usar os métodos de Heaviside. A partir de então, os livros-texto de eletromagnetismo do início do século XX começaram a trazer a teoria de Maxwell tratando o campo elétrico e magnético como grandezas fundamentais do eletromagnetismo e os potencias como grandezas auxiliares destituídas de significado físico, sendo utilizados como artifícios para o cálculo dos campos.

\title{
V. O potencial vetor no século XX e em manuais universitários
}

No início do século XX, pouco se falou a respeito da interpretação física do conceito de potencial vetor em livros-texto ou artigos científicos (ROCHE, 1990; PEREIRA, 2009).

No seu livro The Feynman Lectures on Physics, de 1964, Richard P. Feynman (19181988) discutiu sobre a realidade dos campos e potenciais e não especificamente sobre significados físicos destas grandezas. De acordo com ele, um indício de realidade física para o campo magnético é ele exercer força em uma carga em movimento neste campo. Mas, esta definição, segundo Feynman (1964, p. 15-7), não faz muito sentido, pois:

Nós não sentimos que o campo magnético seja real de alguma forma; nós não podemos, por exemplo, pôr a mão em uma região de campo magnético e senti-lo passando 
através de nossas mãos. Além disso, o valor do campo magnético não é muito definido; é possível escolhermos um sistema de coordenadas movendo-se com certa velocidade, e fazermos com que o campo magnético, em determinado ponto, seja nulo.

Feynman caracterizou o campo como uma definição puramente matemática, na qual um campo real é visto como uma função matemática usada para evitar a ideia de ação à distância. De acordo com ele, um campo real é um conjunto de números que especificamos de tal forma que o que acontece num determinado ponto depende somente de números naquele ponto. Nós não precisamos saber o que está acontecendo em outras partes do sistema.

No caso do potencial vetor, o fato de não ser invariante por escolhas de calibre faz com que ele seja muitas vezes interpretado como não real. No entanto, de acordo com Feynman, "Isto não tem nada a ver com a questão de realidade da forma que é discutida" (FEYNMAN, 1964, p. 15-7). De acordo com o físico estadunidense, é possível alterar o campo magnético, assim como o elétrico e o próprio potencial vetor, para o caso de referenciais que se movem. $\mathrm{O}$ que importa, para Feynman, é discutir se o potencial vetor é real para descrever efeitos magnéticos ou se ele é simplesmente uma ferramenta matemática.

Na década de 1980, o físico estadunidense Emil Jan Konopinski (1911-1990) descreveu um possível significado físico para o conceito de potencial vetor no livro Electromagnetic fields and relativistic particles (KONOPINSKI, 1981). Neste livro, ele considerou que o potencial vetor é o momento do campo disponível para produzir movimento de uma carga $q$ imersa em um campo eletromagnético, chamando-o de momento potencial (KONOPINSKI, 1981, p. 159).

Para ele, é importante enfatizar o significado físico de $\vec{A}$, pois (KONOPINSKI, 1981, p. 159):

A interpretação física do potencial vetor encontrada aqui requer uma ênfase especial porque tem sido persistentemente dito, por alguns autores, que o vetor $\vec{A}$ não possui significado físico. O resultado é que a descrição potencial, por meio de $\phi \vec{e} \vec{A}$, dos campos eletromagnéticos, é tão significativa quanto as descrições por meio de $\vec{E} e$ $\vec{B}$. Enquanto que de $\vec{E}$ e $\vec{B}$ descrevem um campo em termos de forças que o campo pode exercer sobre a matéria carregada, $\phi$ e $\vec{A}$ descrevem o mesmo campo em termos de energia e momento que todo o campo deixa disponível para a matéria.

De acordo com Konopinski, ao colocarmos uma carga $q$ em uma região de campo eletromagnético, a quantidade $(q / c) \vec{A}$ representa o momento do campo disponível para produzir movimento à carga $q$. Da mesma forma que a quantidade $q \phi$ representa a energia potencial, $(q / c) \vec{A}$ serve como o momento potencial para movimentos da carga $q$ (KONOPINSKI, 1981, p. 159).

Em âmbito nacional, um livro digno de nota é o do físico Josif Frenkel de 2006, Princípios de eletrodinâmica clássica, que ao escrever a componente $i$ do momento canônico de uma partícula relativística como $P_{i}=\gamma m v_{i}+(q / c) A_{i}$, diz que: "A parcela $q A_{i} / c$ representa o 
momento armazenado no campo que pode ser transferido à partícula, da mesma forma que $q \phi$ representa a energia potencial da partícula no campo" (FRENKEL, 2006, p. 356). Ou seja, uma interpretação semelhante à de Konopinski, porém num contexto relativístico.

Embora a discussão acerca do significado físico do potencial vetor e dos papeis dos potenciais na teoria eletromagnética seja riquíssima, sob o ponto de vista histórico e do ensino destes conceitos, iniciativas neste sentido são apenas pontuais. A abordagem predominante nos livros mais usados nas universidades brasileiras é de que o potencial vetor é apenas um artifício de cálculo destituído de significado físico. Na tabela 2, selecionamos as posturas adotadas em livros que foram consultados para a construção deste trabalho. Algumas posturas foram discutidas ao longo do texto, outras, consideramos relevante apenas indicar na tabela para futura consulta pelo leitor.

Tabela 2 - Posições de alguns autores de livros-texto sobre o conceito de potencial vetor.

\begin{tabular}{ll}
\hline $\begin{array}{l}\text { Autores que defendem um significado } \\
\text { físico para o potencial vetor }\end{array}$ & $\begin{array}{l}\text { Autores que defendem o uso do potencial } \\
\text { vetor como um simples artifício matemático } \\
\text { ou não discutem um possível significado fí- } \\
\text { sico }\end{array}$ \\
\hline $\begin{array}{l}\text { J.J. Thomson, Electricity and Matter, } \\
1904\end{array}$ & $\begin{array}{l}\text { H. A. Lorentz, Lectures on theoretical physics, } \\
1931\end{array}$ \\
\hline $\begin{array}{l}\text { R. P. Feynman, The Feynman lectures on } \\
\text { physics, 1964 }\end{array}$ & $\begin{array}{l}\text { F. Rohrlich, Classical charged particles: } \\
\text { foundations of their theories, 1965 }\end{array}$ \\
\hline $\begin{array}{ll}\text { E. J. Konopinski, Electromagnetic fields } \\
\text { and relativistic particles, 1981. }\end{array}$ & $\begin{array}{l}\text { Reitz, J. R., Milford, F.; Wentworth, C.R. } \\
1982 .\end{array}$ \\
\hline $\begin{array}{l}\text { J. Frenkel, Princípios de eletrodinâmica } \\
\text { clássica, 2006. }\end{array}$ & $\begin{array}{l}\text { J. D. Jackson, Classical electrodynamics, } \\
1999 .\end{array}$ \\
\hline $\begin{array}{l}\text { E. G. Cullwick, The Fundamentals of } \\
\text { Electromagnetism, 1966. }\end{array}$ & $\begin{array}{l}\text { D. J. Griffits, Introduction to electrodynamics, } \\
1999\end{array}$ \\
\hline
\end{tabular}

Não foi nosso objetivo defender uma ou outra interpretação, mas sim analisar as diferentes posições do ponto de vista histórico mostrando que o desenvolvimento da teoria eletromagnética foi influenciado por fatores teóricos, experimentais, matemáticos, formais, estéticos, práticos, entre outros. Acreditamos que este tipo de discussão pode enriquecer as aulas de eletromagnetismo, contribuir para um melhor entendimento dos conceitos envolvidos na teoria eletromagnética e também mostrar que mesmo uma teoria bem consolidada abriga questões conceituais em aberto. 


\section{Considerações finais}

Neste trabalho, analisamos as múltiplas interpretações dadas ao conceito de potencial vetor no eletromagnetismo clássico desenvolvido na Grã-Bretanha do século XIX. No final do mesmo século, alguns físicos, entre eles, Heaviside e Hertz, reformularam a teoria de Maxwell sintetizando-a em quatro equações fundamentais envolvendo o campo elétrico e magnético e observaram que a simetria destas equações poderia ser interpretada como algo fundamental da teoria eletromagnética. Seus artigos posteriores foram uma campanha pela adoção destas equações mostrando sua aplicabilidade para a solução de diversos problemas práticos e teóricos, antes de difícil tratamento utilizando a abordagem dos potenciais.

A estratégia de Heaviside consistiu na apresentação de suas ideias utilizando principalmente o formalismo vetorial recentemente criado por ele e por Josiah Willard Gibbs (18391903) (SILVA, 2004). Além disso, seu estilo agressivo e incisivo ao defender suas opiniões, contribuiu para a aceitação de suas ideias. Este mesmo tipo de estratégia foi usado por Heaviside, no debate envolvendo o formalismo vetorial e o quaterniônico, também no final do século XIX (SILVA, 2002).

A partir do século XX, os livros-texto de eletromagnetismo começaram a abordar o assunto potencial vetor na forma como é estudado atualmente em grande parte dos cursos de eletromagnetismo: funções auxiliares para o cálculo do campo elétrico e magnético.

Tentamos evitar, ao longo do trabalho, escolher entre uma ou outra postura em relação aos significados atribuídos ao conceito, em vez disso, buscamos mostrar quão rica podem ser as discussões envolvendo a construção dos conhecimentos científicos.

Ao término de um curso de eletromagnetismo, os estudantes de física possuem um entendimento considerável dos campos elétrico e magnético e do potencial escalar. Porém, o entendimento que possuem do conceito de potencial vetor é simplesmente operacional, usandoo apenas como uma grandeza matemática para o cálculo dos campos. Além de importância histórica, temas como este podem contribuir para que professores e estudantes de física tenham noções das diferentes influencias na construção do conhecimento científico, aprofundando a compreensão sobre conceitos abstratos do eletromagnetismo, como é o caso do potencial vetor. Este tipo de discussão poderia ser realizado num curso de eletromagnetismo, pois podem contribuir para que os estudantes adquiram uma compreensão mais profunda de alguns conceitos abstratos do eletromagnetismo e também compreendam melhor como se dá a construção de conceitos científicos.

\section{Referências bibliográficas}

ABRANTES, P. C. C. A filosofia da ciência de H. Hertz (1857-94). In: ÉVORA, F. (Org.). Século XIX: o nascimento da ciência contemporânea. Campinas: Coleção CLE, 1996, p. 351375, v. 11. 
ASSIS, A. K. T. Curso de eletrodinâmica de Weber. Campinas: Instituto de Física Gleb Wataghin, 1992. $140 \mathrm{p}$.

BORK, A. M. Maxwell and the vector potential. Isis, v. 58, n. 2, p. 210-222, 1967.

CULLWICK, E. G. The Fundamentals of Electromagnetism. 3. ed. Cambridge: Cambridge University Press, 1966. 320 p.

DARRIGOL, O. Electrodynamics from Ampere to Einstein. Oxford: Oxford University Press, 2000. 532 p.

FARADAY, M. Experimental researches in electricity. London: Richard and John Edward Taylor, 1839. 622 p, v. 1 .

FEYMAN, R. P. The Feynman lectures on physics. Massachusetts: Addison-Wesley Publishing, 1964. 566 p, v. 2.

FRENKEL, J. Princípios de eletrodinâmica clássica. 2. ed. São Paulo: Edusp, 2006. 416 p.

GRIFFITS, D. J. Introduction to electrodynamics. 3. ed. Upper Saddle River: Prentice Hall, 1999. $576 \mathrm{p}$.

HARMAN, P. M. The scientific letters and papers of James Clerk Maxwell. Cambridge: Cambridge University Press, 1990. 748 p, v. 1.

HEAVISIDE, O. Electrical papers. New York: Chelsea Publishing Company, 1970. v. 1-2.

HEAVISIDE, O. Electromagnetic theory, New York: Chelsea Publishing Company, 1971. v. $1-3$.

HERTZ, H. R. Electric waves. New York: Dover Publications, 1962. 278 p.

HUNT, J. B. Oliver Heaviside: a first-rate oddity. Physics Today, v. 65, n. 11, p. 48-54, 2012. Disponível em: < http://fep.if.usp.br/ fbrandt/fisica4/OliverHeavside.pdf>. Acesso em: 27 abr. 2017.

HUNT, J. B. The maxwellians. 1. ed. Ithaca and London: Cornell University Press, 1991. 280 p.

JACKSON, J. D. Classical electrodynamics. 3. ed. New York: John Wiley \& Sons, 1999. 832 p.

KONOPINSKI, E. J. Electromagnetic fields and relativistic particles. 1. ed. New York: McGraw-Hill, 1981. 159 p.

LORENTZ, H. A. Lectures on theoretical physics. London: The Macmillan Company, 1931. v. 3, 47 p. 
LORENZO, J. A.; DONCEL, M. G. The electrotonic state, a metaphysical device for Maxwell too? European Journal of Physics, v. 17, n.1, p. 6-10, 1995.

MARTINS, R. A. Oersted e a descoberta do eletromagnetismo. Cadernos de História e Filosofia da Ciência, n. 10, p. 89 - 114, 1986. Disponível em: < http://www.ghtc.usp.br/server/pdf/ram-30.pdf>. Acesso em: 27 abr. 2017.

MAXWELL, J. C. A treatise on electricity and magnetism. 3. ed. New York: Dover, 1954. 577 p, v. 1, 532 p, v. 2.

MAXWELL, J. C. The scientific papers of James Clerk Maxwell. 1. ed. New York: Dover, 1965. 668 p, v. 1, 829, v. 2.

MCCORMMACH, R. Heinrich Rudolf Hertz. In: SCRIBNER, C. (Org.). Dicitionary of scientific biography. New York: Charles Scribner's Sons, 1981, p. 340-350, v. 6.

O'HARA, J. G.; PRICHA, W. Hertz and the Maxwellians: A study and documentation of the discovery of electromagnetic wave radiation, 1873-1894. 1. ed. London: Peter Peregrinus, 1987. $154 \mathrm{p}$.

PEREIRA, A. G. Um estudo histórico da evolução do conceito de potencial vetor no eletromagnetismo clássico. 2009. 134 f. Dissertação (Mestrado em Física) - Instituto de Física de São Carlos, Universidade de São Paulo, São Carlos.

POYNTING, J. H. On the transfer of energy in the electromagnetic field. Philosophical Transactions of the Royal Society of London, v. 175, p. 343-361, 1884.

REITZ, J. R. Fundamentos da teoria eletromagnética. 1. ed. Rio de Janeiro: Campus, 1982. $459 \mathrm{p}$.

ROCHE, J. J. A critical study of the vector potential. In: ROCHE, J. J. Physicists look back: studies in the history of physics. Oxford: Oxford Linacre College, 1990. p. 144-168.

ROHRLICH, F. Classical charged particles: foundations of their theories. 1. ed. New York: Addison-Wesley, 1965. 305 p.

SILVA, C. C. Da força ao tensor: evolução do conceito físico e da representação matemática do campo eletromagnético. 2002. 250 f. Tese (Doutorado em Física) - Instituto de Física Gleb Wataguin, Universidade Estadual de Campinas, Campinas.

The role of models and analogies in the electromagnetic theory: a historic case study. Science \& Education, v. 16, n. 7-8, p. 835-848, 2007.

A escolha de uma ferramenta matemática para a física: o debate entre quaternions e a álgebra vetorial de Gibbs e Heaviside. In: MARTINS, R. A.; MARTINS, L. A. C. P; 
SILVA, C. C; FERREIRA, J. M. H. (Org.). Filosofia e História da Ciência no Cone Sul: $3^{o}$ Encontro. Campinas: AFHIC, 2004. p. 115-126.

; MARTINS, R. A. Polar and axial vectors versus quaternions. American Journal of Physics, v. 70, p. 958-63, 2002.

THOMSON, J. J. Electricity and matter. London: Constable and Company, 1911.

THOMSON, W. A mathematical theory of Magnetism. Philosophical Transactions of the Royal Society of London, v. 141, p. 243-285, 1851.

WHITTAKER, S. E. A history of the theories of aether and electricity. New York: Humanities Press, 1973. v.1.

WISE, N. M. The mutual embrace of electricity and magnetism. Science, v. 203, n. 4387, p. 1310-1318, 1979. 